\title{
Maturation, pharmacogenomics and metabolomics as factors determining pharmacokinetic and pharmacodynamics profile of alpha-agonist in pediatric intensive care unit patients
}

\author{
Joanna Bartkowiak-Wieczorek', Justyna Mocarska', Alicja Bartkowska-Śniatkowska², \\ Jan Matysiak ${ }^{3}$, Agnieszka Klupczyńska ${ }^{3}$, Zenon J. Kokot ${ }^{3}$, Paweł Wiczling ${ }^{4}$, \\ Edmund Grześkowiak', Agnieszka Bienert ${ }^{1}$ \\ ${ }^{1}$ Department of Clinical Pharmacy and Biopharmacy, Poznan University of Medical Sciences, Poland \\ ${ }^{2}$ Department of Pediatric Anesthesiology and Intensive Therapy, Poznan University of Medical Sciences, Poland \\ ${ }^{3}$ Department of Inorganic and Analytical Chemistry, Poznan University of Medical Sciences, Poland \\ ${ }^{4}$ Department of Biopharmaceutics and Pharmacodynamics, Medical University of Gdańsk, Poland
}

\begin{abstract}
Research Project Objectives. Project entitled "Maturation, pharmacogenomics, and metabolomics as factors determining pharmacokinetic and pharmacodynamic profile of alpha-agonist in pediatric intensive care unit patients" was founded by the Polish National Science Center (NCN) under project number: 2015/17/B/NZ7/03032. The duration of the grant is 36 months, and the total grant value is 688800 PLN. The project is run by the Medical University of Gdansk and Poznan University of Medical Sciences. The aim of this grant is to examine the influence of maturation, pharmacogenetics, metabolomics and physiological (or pathophysiological) status of the patients on the pharmacokinetics and pharmacodynamics (PK/PD) of $\alpha 2$-adrenergic drugs (dexmedetomidine and clonidine) in pediatric population. The project was proposed to explain the unusual PK of dexmedetomidine reported in literature and in our preliminary experiments, in which a two-fold increase in dexmedetomidine clearance was observed during prolonged (lasting more than $24 \mathrm{hr}$ ) infusions in the intensive care unit patients. General information. Project entitled "Maturation, pharmacogenomics, and metabolomics as factors determining pharmacokinetic and pharmacodynamics profile of alpha-agonist in pediatric intensive care unit patients" was founded by the Polish National Science Center (NCN) under project number: 2015/17/B/NZ7/03032. The duration of the grant is 36 months, from 2016-04-27 to 2019-04-26 and the total grant value is 688800 PLN. The project is run by the Medical University of Gdansk and Poznan University of Medical Sciences. The research group consists of: principal investigator dr hab. Paweł Wiczling and co-investigators: dr hab. Agnieszka Bienert, dr Alicja Bartkowska-Śniatkowska, dr Joanna Bartkowiak-Wieczorek, mgr Justyna Mocarska, prof. Edmund Grześkowiak, dr Jan Matysiak, mgr Agnieszka Klupczyńska, dr Danuta Siluk, mgr Agnieszka Borsuk and prof. dr hab. Zenon J. Kokot. The Ethical Committee permission number is 261/15.
\end{abstract}

Keywords: pharmacogenomics, metabolomics, pharmacokinetic, pharmacodynamics, dexmedetomidine, clonidine.

\section{Research plan}

The project will be implemented in three steps:

1. The first research task will include collection of the required experimental data from the patients: arterial blood samples, the values of two sedation scores (Ramsay scale and Glasgow Coma Scale modified by Cook and Palma (GSCS) scale), blood pressure, heart rate, body temperature, cardiac 
output, blood oxygen saturation, biochemical and demographic parameters. Pediatric risk of mortality (PRISM), as well as Pediatric Multiple Organ Dysfunction Score (P-MODS) will be used to characterize the health status.

2. The second research task will cover: 1) development of sensitive and reliable analytical methods (LC-MS/ MS) applicable for quantitative determination of clonidine and dexmedetomidine; 2) untargeted (exploratory) and targeted metabolomics analysis. The exploratory phase will focus on the analysis of the whole metabolic fingerprints in plasma samples without distinction of the particular compounds or group of analytes. It will be followed by the targeted approach which will be focused on dexmedetomidine and clonidine metabolites and other compounds indentified during the exploratory phase; and 3) the pharmacogenomic analysis to determine the frequency of particular gene polymorphisms such as ADRA2B, ADRA2A, UGT, CYP2A6 affecting the pharmacokinetic and pharmacodynamic processes of the studied drugs.

3. The third research task will involve the development of a population (hierarchical) PK/PD model to describe the drug concentrations and effects (Ramsay scale and Glasgow Coma Scale) time-course, to quantitate inter-individual variability and to find factors significantly affecting the PK/PD profiles of the studied drugs through the covariance analysis.

\section{Basic Concept}

Individualization of pharmacotherapy plays a crucial role in the treatment, especially among PICU patients. In that population the differences in drug response might be associated with various demographic or physiological parameters, i.e. age, metabolizing enzyme activity and genetic variability of enzymatic pathways [8]. The main objective of our study is to elucidate the mechanism responsible for the time-dependent increase in dexmedetomidine clearance observed in PICU patients during infusion longer than $24 \mathrm{hr}$. We hypothesized that this effect depends on the genetic variability of metabolizing enzymes and $\alpha 2$-adrenergic drugs receptors. However, another explanation are also possible, i.e. through changes in patients' cardiac output during the long-term infusion. The patients' health status can change during hospitalization and as a result the drug clearance might change for high/ moderate hepatic extraction ratio drugs, such as dexmedetomidine. In order to verify these hypotheses we are going to analyze the influence of maturation (age), pharmacogenetics (genetic variability), metabolomics (difference in metabolic profiles), and physiological (or pathophysiological) status of the patients on the pharmacokinetics and pharmacodynamics (PK/PD) of two $\alpha 2$-adrenergic drugs using the state-of-the-art methodology (nonlinear mixed-effect modeling).

\section{Research Methodology}

Patients will be included in the study based on the prespecified inclusion and exclusion criteria. Dexmedetomidine and clonidine will be administered as intravenous infusion through microbore tubing into a central catheter with the initial dosing of $0.8 \mathrm{mcg} / \mathrm{kg}$ b.w./hr and $1 \mathrm{mcg} / \mathrm{kg}$ b.w./hr, respectively. Drugs will be delivered based on the level of sedation up to a maximum rate of $1.4 \mathrm{mcg} / \mathrm{kg}$ b.w./hr for dexmedetomidine and 2 $\mathrm{mcg} / \mathrm{kg}$ b.w./hr for clonidine.

The sedation monitoring will be provided by the use of the Glasgow Coma Scale modified by Cook and Palma (GSCS) scale, Comfort Scale and the Ramsay score. The GSCS and Comfort Scale have previously been validated as a sedation scale for mechanically ventilated patients [9]. Arterial blood samples (2 ml) will be obtained before administration of the loading dose, during the infusion of dexmedetomidine or clonidine, and after infusion cessation.

\section{Experimental design}

The optimization and simulations methods will be used to evaluate the experimental design. Population study (i.e. the total number of subjects to be studied, number of groups and number of individuals per group, dosing and sampling schedules) will be established before performing a prospective analysis with a parametric method (such as NONMEM) in order to ensure the best precision of the population parameter estimates. The population studies design will be based on a free software programs as POPed version 2.13 (http://poped. sourceforge.net/index.php).

\section{Dexmedetomidine and clonidine quantification}

Extraction of the studied compounds from plasma samples will be performed with the use of solid-phase extraction technique. Thawed samples at a volume of 200-300 $\mu \mathrm{l}$ will be used. SPE Plexa cartridges (30 mg, $1 \mathrm{ml}$, Agilent Technologies, Palo Alto, CA, USA) will be employed in order to extract the analytes. The solvent mixtures composition and their volumes, necessary for efficient SPE processing, will be determined 
during the final method optimization. Extracted samples will be evaporated to dryness at a miVac Quattro Sample Concentrator (Genevac, Suffolk, UK), reconstituted with $100 \mu \mathrm{l}$ of the mobile phase, and injected into the chromatographic system. Analyses will be performed using an HPLC system (Agilent Technologies). The mobile phase, pumped at a flow rate of $\sim 0.3 \mathrm{ml} /$ min, will be composed of a mixture of methanol and water with 0.1 formic acid. The optimal temperature for the separation (column compartment temperature) will be tested while the autosampler will be thermostated at $4^{\circ} \mathrm{C}$.

\section{Metabolomics}

The plasma samples will be analyzed with high performance liquid chromatography coupled with time of flight mass spectrometry (HPLC-TOF-MS, Agilent Technologies), gas chromatography hyphenated to triple quadrupole mass spectrometry (GC-MS/MS, Shimadzu) as well as capillary electrophoresis coupled with the time-of-flight mass spectrometry (CE-TOF-MS, Agilent Technologies). To extract the information on putative biomarkers, the data sets will subjected to for chemometric preprocessing methods, such as peak alignment, filtering and normalization $[4,5]$. Subsequently, the normalized data will be analyzed using multivariate statistical analysis (multilevel regression models) in order to indentify the metabolites of studied drugs and endogenous compounds with unusual (in comparison to the mean behavior) time-courses. The preprocessing methods will be performed with the use of Mass Profiler Professional Software (Agilent Technologies). The regression will be performed using MATLAB 9.1 (The MathWorks, Inc., U.S.A.) $[5,6]$. For the identification of unknown metabolites, the scientific databases will be used.

The second analytical approach in this part will be a targeted metabolomic analysis. The analytical methods will use a triple quadrupole mass spectrometry detection coupled with high performance liquid chromatography technique (HPLC-MS/MS), capillary electrophoresis (CE-MS/MS) and gas chromatography (GC-MS/MS). In targeted analysis we will use multiple reaction monitoring mode (MRM mode) to determine the known compounds (dexmedetomidine and clonidine metabolites). The obtained data sets will be analyzed using multilevel regression modeling.

\section{Pharmacogenomics}

The presence of gene polymorphism in ADRA2B, ADRA2A, UGT, CYP2A6 will be established. Genomic
DNA will be isolated from whole blood using "QiAamp DNA Blood Mini Kit" (Qiagen). Genotyping will be performed by real-time PCR method using probes or PCR-RFLP method (polymerase chain reaction and restriction analysis). Reactions of real-time PCR will performed with the use of LightCycler ${ }^{\circledR 480}$.

Pharmacokinetics and pharmacodynamics The concentrations of dexmedetomidine and clonidine and clinical endpoints will be described by a population pharmacokinetic and pharmacodynamic model [7]. Population nonlinear mixed-effect modeling will be done using NONMEM (Version 7.2.0, Icon Development Solutions, Ellicott City, MD, USA) and the gfortran compiler 9.0. NONMEM runs will be executed using Wings for NONMEM (WFN720, http:// wfn.sourceforge.net). The first-order conditional estimation with interaction (FOCE) method will be used. The self-written differential equations will be used as model equations. The NONMEM data processing and plots will be done in Matlab ${ }^{\circledR}$ Software version 9.1 (The MathWorks, Inc., Natick, MA, USA). The minimum value of the NONMEM objective function, typical goodness-of-fit diagnostic plots, and the evaluation of the precision of PK/PD parameter and variability estimates will be used to discriminate between various models during the model-building process. The hypothesis testing (i.e. covariance analysis) will be based on a minimum value of objective function (MOF). The difference in MOF of 10.8 for one degree of freedom and 13.8 for two degrees of freedom between two hierarchical models will be considered statistically significant at $p<0.001$. The visual predictive checks will be performed to asses each model predictive performance. The uncertainty of all PK/PD parameters will be obtained from the non-parametric bootstrap method.

\section{Measurable Effects}

To our knowledge it is the first such a comprehensive study in the field of PK/PD modeling in patients from the pediatric intensive care unit. In our opinion the findings of this project may extend the fundamental knowledge of pharmacokinetics and pharmacodynamics of a2-adrenergic drugs and quantitate the pharmacokinetic and pharmacodynamic differences among patients from special population, i.e. children in intensive care units. This may be further used to improve the PK/PD models and the drug dosing regiments. 


\section{Expected Results}

The results obtained in this project will allow for a thorough examination of the dynamics of CYP2D6 and CYP2A6 enzymes maturation and its effect on the clonidine and dexmedetomidine metabolism. In this are the number of reported studies is limited and the current literature results do not clearly address the questions concerning the degree of variability of metabolism of both drugs. Therefore, it seems to be important to search for association between polymorphic variation and the level of mRNA expression of selected metabolic enzymes for clonidine and dexmedetomidine and clinical parameters, especially in children and newborns. In our opinion it is also worth to study the metabolomic profile of the patient as the metabolites concentration should also manifest clinically relevant inter-individual and intra-individual differences. The results of the project will provide a rationale for the dose adjustments and might increase the safety of sedation in children at different ages and under different pathophysiological conditions.

\section{Acknowledgements}

\section{Conflict of interest statement}

The authors declare no conflict of interest.

\section{Funding sources}

The work is a part of the project "Maturation, pharmacogenomics, and metabolomics as factors determining pharmacokinetic and pharmacodynamic profile of alpha-agonist in pediatric intensive care unit patients", supported by the Polish National Science Center under grunt number 2015/17/B/ NZ7/03032.

\section{References}

1. Clayton TA, Lindon JC, Cloarec O, Antti H, Charuel C, Hanton G, Provost JP, Le Net JL, Baker D, Walley RJ, Everett JR, Nicholson JK. Pharmaco-metabonomic phenotyping and personalized drug treatment. Nature. 2006 Apr;440(7087):1073-1077.
2. Nebert DW, Vesell ES. Advances in pharmacogenomics and individualized drug therapy: exciting challenges that lie ahead. Eur J Pharmacol. 2004;500(1-3):267-280.

3. lirola $T$, Ihmsen $H$, Laitio $R$, Kentala $E$, Aantaa R, Kurvinen JP, Scheinin $\mathrm{M}$, Schwilden $\mathrm{H}$, Schüttler J, Olkkola KT. Population pharmacokinetics of dexmedetomidine during long-term sedation in intensive care patients. $\mathrm{Br} J$ Anaesth. 2012 Mar;108(3):460-468.

4. Struck W, Wiczling P, Waszczuk-Jankowska M, Kaliszan R, Markuszewski MJ. New supervised alignment method as a preprocessing tool for chromatographic data in metabolomic studies. J Chromatogr A. 2012 Sep;1256:150-159.

5. Struck W, Siluk D, Yumba-Mpanga A, Markuszewski M, Kaliszan R, Markuszewski MJ. Liquid chromatography tandem mass spectrometry study of urinary nucleosides as potential cancer markers. J Chromatogr A. 2013 Mar;1283:122-131.

6. Struck W, Waszczuk-Jankowska M, Kaliszan R, Markuszewski MJ. The state-of-the-art determination of urinary nucleosides using chromatographic techniques "hyphenated" with advanced bioinformatic methods. Anal Bioanal Chem. 2011 Oct;401(7):2039-2050.

7. NONMEM Users Guides. In: Beal SL, Sheiner LB, editors. Maryland: Icon Development Solutions; 1989-1998.

8. Bienert A, Płotek W, Wiczling P, Warzybok J, Borowska K, Buda K, Kulińska K, Billert H, Kaliszan R, Grześkowiak $E$. The influence of age and dosage on the pharmacodynamics of dexmedetomidine in rabbits. JMS. 2014;83(2):108-115.

9. Carrasco G. Instruments for monitoring intensive care unit sedation. Crit Care. 2000;4:217-225.

Acceptance for editing: 2016-06-10 Acceptance for publication: 2016-06-23

\section{Correspondence address:} Justyna Mocarska

Department of Clinical Pharmacy and Biopharmacy Poznan University of Medical Sciences, Poland Św. Marii Magdaleny 14 Street, 61-861 Poznań, Poland phone: +486166878 37 fax: +48616687855 email: justyna.mocarska@gmail.com 